\title{
O que Dizem os Pacientes dos Serviços Privados de Radiologia, Portugal
}

\section{What do the Patients Say about the Radiology Private Services, Portugal}

\author{
Carla Regina Rodrigues Guerra Silva \\ Técnica de Radiologia no Centro Hospitalar de Trás-os-Montes e \\ Alto Douro Unidade de Vila Real, Portugal. Mestre em Gestão dos \\ Serviços de Saúde. \\ Endereço: Avenida da Noruega, Lordelo, 5000-508 Vila Real, \\ Portugal. \\ E-mail: creginaœsapo.pt \\ Vitor Manuel Costa Pereira Rodrigues \\ Professor Coordenador (PhD) com Agregação na ESEnf. da Uni- \\ versidade de Trás-os-Montes e Alto Douro - Portugal. Membro \\ Integrado do CIDESD (Centro de Investigação em Desporto, Saúde \\ e Desenvolvimento Humano da UTAD. Doutorado em Ciências Bio- \\ médicas. É um dos Directores do Mestrado em Gestão dos Serviços \\ de Saúde da UTAD. Linhas de Investigação: Sistemas e serviços de \\ saúde; Promoção da saúde ao longo do ciclo vital. \\ Endereço: Lugar do Tojal, Lordelo, 5000-232 Vila Real, Portugal. \\ E-mail: vmcpr®utad.pt
}

\section{Resumo}

Objectivo: A satisfação do cliente tem vindo a afirmar-se como uma das áreas prioritárias na avaliação da qualidade nos cuidados de saúde. O principal objectivo desta investigação é conhecer o nível de satisfação dos pacientes dos serviços privados de radiologia. Materiais e Métodos: Tratou-se de um estudo exploratório e descritivo numa amostra de 213 pacientes do Conselho de Vila Real (Portugal). Resultados: A maior parte dos pacientes efectuou exames de mamografia; mais de $50 \%$ dos inquiridos esperou mais de uma semana desde o dia da marcação até ao dia em que ia efectuar o exame. Os inquiridos encontram-se satisfeitos com a qualidade da maioria dos serviços prestados, expressando vontade em voltar e recomendar os serviços a outras pessoas. Referiram a existência de lacunas na relação e comunicação, organização dos serviços, informação e apoio e organização do espaço físico. Conclusão: Este estudo é importante na monitoração e garantia da qualidade e para expressar a percepção dos clientes.

Palavras-chave: Radiologia; Satisfação do Paciente; Qualidade da Assistência à Saúde. 


\section{Abstract}

Objective: Patients' satisfaction is now one of the most relevant aspects when evaluating the quality of healthcare services. The purpose of this study was to know the users' satisfaction levels in radiology private services. Materials and Methods: Our empirical research followed an exploratory and descriptive approach. It reflects a sample of 213 patients in Vila Real (Portugal). Results: The majority of patients who came to our services had mammographies done; over $50 \%$ of the interviewees claimed having had to wait more than a week to get an appointment. The users have expressed their wish to return, to recommend the services to other people, and also mentioned the existence of a few weak points in terms of communication, organization, information and support provided. Conclusion: This study was important because it allowed us to become aware of the users' points of view and also of any possible improvement points in terms of the quality of the service provided.

Keywords: Radiology; Patient Satisfaction; Quality of Health Care.

\section{Introdução}

A necessidade constante de melhorar a qualidade nos serviços, como os de assistência na saúde, que incidem na qualidade de vida e no futuro dos cidadãos, é hoje, cada vez mais, um imperativo inadiável para as empresas e outras organizações responsáveis pela prestação desses serviços. Actualmente, as organizações de serviços na área de saúde, públicas e privadas, que não visam à gestão da qualidade, acabam por empregar os seus recursos de forma ineficiente e ineficaz, não só influenciando os seus resultados, como afectando até mesmo a sua sobrevivência no mercado (Sakellarides e col., 2006).

O Sistema de Saúde Português, ou Serviço Nacional de Saúde (SNS), envolve todos os cuidados integrados de saúde, desde a promoção e vigilância da saúde, a prevenção da doença, o diagnóstico e tratamento e reabilitação dos doentes. Goza de autonomia administrativa e financeira, estrutura-se numa organização descentralizada e desconcentrada (Gonçalves e Costa, 2003), compreendendo órgãos de âmbito central (Ministério da Saúde), regional (Subregiões de Saúde) e local, e dispõe de serviços prestadores de cuidados de saúde primários (Centros de Saúde) e serviços prestadores de cuidados de saúde diferenciados (Hospitais).

O SNS foi regulado, a partir de 1990, por dois diplomas fundamentais: a Lei de Bases da Saúde (Lei $\mathrm{n}^{0}$ 48/9o, de 24 de Agosto) e o Estatuto do Serviço Nacional de Saúde (Lei no 22/93, de 15 de Janeiro). Para muitos, o ano de 1990 é considerado como um ano de viragem decisiva no Sistema de Saúde Português (Sepúlveda, 1998).

O actual Sistema de Saúde Português é "um grande espaço de convergência e congregação de esforços", composto por "três segmentos claramente individualizados" e articulados entre si, que são consequência da evolução do sistema de saúde nos últimos trinta anos (Almeida, 1999):

i) o SNS, abrangendo todas as instituições e serviços oficiais prestadores de cuidados de saúde dependentes do Ministério da Saúde;

ii) todas as entidades públicas que desenvolvem actividades de promoção, prevenção e tratamento na área da saúde;

iii) todas as entidades privadas e todos os profissio- 
nais livres que acordem com o SNS a prestação de cuidados ou de actividades de saúde.

É preciso dizer que o Sistema Nacional de Saúde é um conjunto ordenado e hierarquizado de instituições e de serviços oficiais prestadores de cuidados de saúde, funcionando sob a tutela do Ministério da Saúde. A sua organização é descentralizada através das Regiões/Sub-Regiões de Saúde, que estabelecem o contacto entre o nível local e o Ministério da Saúde. Existem cinco Regiões de Saúde: Norte, com sede no Porto; Centro, com sede em Coimbra, Lisboa e Vale do Tejo, com sede em Lisboa; Alentejo, com sede em Évora e Algarve, com sede em Faro. Existem ainda Agrupamentos de Centros de Saúde (ACES) que vieram substituir as 18 sub-regiões, correspondentes a cada um dos Distritos do Continente.

Em cada Região/Sub-Região de Saúde, a prestação de cuidados de saúde é assegurada, pelos Hospitais e pelos Centros de Saúde.

Falando de Vila Real e do seu conselho, podemos dizer que Vila Real é a capital do Distrito, encontra-se situada na Região Norte e concretamente na região do Douro. É um conselho que tem cerca de 50. ooo habitantes e está subdividido em 30 freguesias.

Quanto à prestação de cuidados de saúde no conselho de Vila Real, podemos referir que existe o Centro Hospitalar de Trás-os-Montes e Alto Douro, que é um Hospital integrado na Administração Regional de Saúde do Norte, com área de influência abrangendo cerca de 300.00o habitantes dos conselhos de: Tarouca, Tabuaço, S. João da Pesqueira, Montalegre, Lamego, Chaves, Boticas, Armamar, Vila Real, Vila Pouca de Aguiar, Santa Marta de Penaguião, Sabrosa, Ribeira de Pena, Peso da Régua, Murça, Mondim de Basto, Mesão Frio e Alijó. O hospital apresenta todas as valências de um hospital central, quer nas áreas médicas, quer nas áreas cirúrgicas, quer em todos os serviços de apoio, tal como o Hospital de Dia e a Imagiologia.

Relativamente aos cuidados de Saúde Primários, existem 2 Centros de Saúde, que funcionam de segunda-feira a sexta-feira das o8:0o às 20:0o horas, e pertencem ao Agrupamento de Centros de Saúde Douro I - Marão e Douro Norte: o Centro de Saúde Vila Real I (que tem 2 extensões em duas localidades) e o Centro de Saúde Vila Real II (que também tem 2 extensões em outras duas localidades e uma unidade de Saúde Familiar).

No que diz respeito a serviços privados de imagiologia, o conselho de Vila Real é servido por três instituições que prestam serviços na área.

Hoje em dia, pode dizer-se que o que caracteriza um bom sistema de saúde é a combinação de quatro factores: uma boa qualidade, um grau de satisfação razoável por parte dos utentes, custos controlados e um sistema de financiamento repartido, até porque as necessidades de saúde da população vão exigir do sistema de saúde respostas cada vez mais complexas, por força de uma progressiva alteração do perfil epidemiológico das doenças e dos doentes cada vez mais envelhecidos e com patologias mais incapacitantes e onerosas (Pinto, 2003).

0 recurso aos serviços de entidades privadas para a obtenção do diagnóstico da doença torna-se inevitável. Apesar da criação do Serviço Nacional de Saúde (SNS), sempre existiram limitações e estrangulamentos na oferta do sector público, tendo o sector privado funcionado como forma de atenuar essas tensões. As organizações privadas são, como qualquer outra organização, susceptíveis de concorrência de mercado e, por isso, necessitam de melhorias contínuas e potenciadoras de desenvolvimento social e económico. Com o crescente nível de competitividade no mercado, pode-se inferir que a retenção de clientes é fundamental e que o grande desafio passa a ser o de reconhecer os clientes, mostrando-lhes o quanto a entidade os estima por lhe terem conferido a sua preferência (Santana, 2005). A retenção de clientes é a chave para o sucesso no mercado do novo milénio e o componente mais importante para a consolidação da participação de mercado da empresa, sendo direccionada, basicamente, pela satisfação do cliente (Vavra e Pruden, 1995). Actualmente, os serviços de saúde desenvolvem estratégias para se diferenciarem porque os clientes têm um maior nível de exigência em relação à personalização de serviços, esperando que se reconheçam as suas necessidades específicas, os seus valores e que a oferta seja adequada aos mesmos. Enfim, requer-se que os serviços de saúde tenham uma maior orientação para o cliente. A perspectiva do utente é considerada indispensável, tanto para monitorizar a qualidade dos serviços de saúde, como para identificar problemas a corrigir, expectativas 
em relação aos cuidados e, ainda, reorganizar os serviços de saúde (Mezomo, 2001).

Foi a partir da Lei de Bases da Saúde (Lei n ${ }^{\circ}$ 48/9o, de 24 de Agosto) e do Estatuto do Serviço Nacional de Saúde (Lei no 22/93, de 15 de Janeiro), que se instituiu o princípio da responsabilização conjunta dos cidadãos, da sociedade e do Estado, na liberdade de procura e de prestação de cuidados (Barros, 2005).

Com efeito, a existência conjunta de prestadores públicos e privados é definitivamente reconhecida na Lei de Bases da Saúde justificando que o SNS não preste a totalidade dos cuidados. Podemos referir que $76 \%$ da população portuguesa tem como único pagador o SNS, e os restantes $24 \%$ dos portugueses utilizam outros subsistemas, dos quais, por exemplo, a ADSE (Assistência na Doença aos Servidores Civis do Estado) abrange 14\% da população (Escoval, 1999; Santana, 2005).

Os subsistemas funcionam como um sistema de saúde em que uma fracção considerável da população beneficia de mecanismos de protecção em situação de necessidade de cuidados médicos para além do SNS. O maior desses sistemas adicionais de protecção é a ADSE, que cobre os funcionários públicos.

Existem actualmente 16 subsistemas de saúde, sendo responsáveis pelo financiamento/prestação de cuidados de saúde a cerca de $24 \%$ da população. Os principais subsistemas públicos de saúde são a ADSE (Assistência na Doença aos Servidores Civis do Estado), os SSMJ (Serviços do Ministério da Justiça), a ADMA (Assistência à Doença Militares da Armada), a ADFA (Assistência à Doença dos Militares da Força Aérea), a ADME (Assistência à Doença dos Militares do Exército) os SAD/PSP (Serviços de Assistência à Doença da Policia de Segurança Pública) e os SAD/GNR (Serviços de Assistência à Doença à Guarda Nacional Republicana).

A reforma do sistema de saúde parte da premissa de que o aumento da concorrência entre serviços prestadores de cuidados de saúde irá crias pressões competitivas, tais como num mercado, de forma a promover uma melhor utilização de recursos e a proporcionar melhores serviços para os cidadãos. As forças da concorrência são o agente de mudança que irão conduzir à eficiência do sistema (Nunes,
2005; Ferreira e Raposo, 2006).

Espera-se que, com a liberdade de escolha por parte dos cidadãos, existam pressões do lado da procura no sentido da maior qualidade dos serviços prestados. Na verdade, os actuais utentes dos sistemas de saúde apresentam características que diferem substancialmente daquelas que existiam aquando da criação do SNS: a subordinação cultural dos doentes é menor. Actualmente, os cidadãos e utentes dispõem de mais capacidades e recursos. Eles são mais exigentes, o que certamente terá um efeito no relacionamento com as instituições de saúde públicas e privadas (Rosa, 2006).

Os novos sistemas de financiamento que vêm sendo introduzidos na saúde levam ao fim da gratuitidade universal. O sistema público passa a fazer parcerias com serviços e entidades privadas incentivando uma competição gerida; justificando que o SNS não preste a totalidade dos cuidados. Assim, os beneficiários utilizam serviços privados convencionados como sejam consultas médicas e meios complementares de diagnóstico e terapêutica. O Serviço Nacional de Saúde tem dificuldades de funcionamento porque a "máquina é muito grande" e torna-se difícil controlar o desperdício, aparecendo o serviço privado como uma boa alternativa ao SNS. De acordo com Barros (2005) e Rocha (2006), o sector privado em nível da imagiologia representa 72,6\% do total de pessoas que realizaram ecografias e $44,0 \%$ raios x. Estima-se que, em 2006, o SNS tivesse gasto em meios complementares de diagnóstico, pagos a privados, cerca de 681 milhões de euros.

0 recurso aos serviços privados de saúde tem vindo a aumentar, esperando-se que, com a liberdade de escolha por parte dos cidadãos, existam pressões do lado da procura no sentido da maior qualidade dos serviços prestados (Hespanhol, 2004).

É um facto que o Estado, ao estabelecer convenções com o sector privado e ao reduzir o investimento no sector público, começa "a comprar" serviços de saúde a fornecedores do sector privado, o que implica o aparecimento de novas unidades de saúde e de fornecimento de serviços privados.

É necessário dizer, também, que a cobertura que os serviços de prestação de cuidados de saúde privados, sejam hospitais, clínicas médicas, laboratórios de análises clínicas e serviços de imagiologia, têm 
em Portugal não é homogénea, uma vez que estes serviços situam-se preferencialmente nas grandes cidades e, sobretudo, em zonas do litoral (onde existe mais população e com um poder económico muito superior ao da população do interior). Existem cidades como Porto, Lisboa e Coimbra que têm 2 ou 3 hospitais privados e dezenas de laboratórios de análises clínicas e serviços de imagiologia privados, enquanto outras cidades, nomeadamente do interior, como por exemplo Bragança, Vila Real, Lamego e Chaves não têm qualquer hospital privado e têm em média 2 ou 3 serviços privados de análises clínicas e de imagiologia.

É também um facto que, neste início do século XXI, o SNS continua a enfrentar graves problemas, tais como (Simões e col., 2009): i) listas de espera longas, para procedimentos cirúrgicos e exames complementares de diagnóstico; ii) aumento das despesas de saúde e dificuldades no controlo dos custos; iii) aumento da procura de cuidados de saúde, sobretudo por parte de grupos vulneráveis.

Efectivamente, e no que diz respeito à prestação de cuidados de saúde, o SNS consiste numa rede de prestadores públicos e privados de saúde, mas cada um relacionado com o Ministério da Saúde. Contudo, o SNS tem permitido, aos seus utentes, oportunidades de poder usufruir da componente de prestação privada, a qual é financiada pelo sector público. Neste caso, podemos referir, desde logo, o fornecimento de alguns medicamentos, sobretudo para patologias crónicas, a hemodiálise e a realização de diversos exames complementares de diagnóstico e terapêutica.

O SNS presta, predominantemente, cuidados de saúde em âmbito hospitalar e cuidados de saúde primários. O sector privado assume a maioria dos serviços prestados no âmbito de consultas das várias especialidades médicas; serviços de diagnóstico (concretamente os prestados no âmbito da imagiologia e análises clínicas); diálise renal e tratamentos de fisioterapia, sendo que estes serviços são prestados pelo sector privado, sob disposições contratuais com o SNS, seja através da contratualização, seja através da comparticipação dos diversos subsistemas de saúde, ou mesmo até através de seguros de saúde (Simões e col., 2007).

\section{Materiais e Métodos}

Este é um estudo exploratório e descritivo. A população é composta pelos pacientes que frequentam as 3 instituições privadas na área da Imagiologia do conselho de Vila Real (Portugal). A amostra foi constituída por 213 pacientes que se deslocaram às terças - feiras, no período da manhã e tarde e sábados, no período da manhã, aos referidos serviços nos meses de Março e Abril de 2007.

Para a recolha de dados, utilizámos um formulário estruturado em 4 grupos: o primeiro grupo é constituído por três questões, destinadas à caracterização da amostra: sexo; idade e grau de instrução; o segundo grupo é constituído por sete questões com a finalidade de obter dados sobre o serviço de Imagiologia no que respeita: ao tipo de exame; às vezes que o cliente lá esteve; como teve conhecimento sobre o serviço; quem fez a marcação do exame; tempo que demorou a saber o dia do exame; tempo que decorreu desde a marcação até ao dia do exame e o tipo de organismo utilizado para a realização desse exame; o terceiro grupo tem a ver com as dimensões que traduzem o nível de satisfação do cliente: Atendimento (todo o processo/procedimentos necessários à recepção do cliente no consultório), Dimensão Funcional Técnica e Médica (conjunto de actividades desenvolvidas pelos profissionais de saúde, e engloba os elementos humanos dos cuidados e as relações sociais e psicológicas entre o paciente e os prestadores de cuidados, as explicações acerca da doença e do tratamento assim como informação recebida) e Amenidades (atributos que podem contribuir para diminuir o desconforto ou a adversidade de um meio estranho: condições da sala de espera, limpeza, estrutura física do serviço e condições das instalações sanitárias); o quarto grupo é constituído por: 3 questões fechadas, sendo que duas se referem à intenção de voltar ao serviço e recomendá-lo a amigos/familiares e uma outra questiona se foi dada alguma informação em como reclamar e quatro questões abertas, nas quais os clientes podem dar a sua opinião sobre: as razões porque voltariam ou não ao serviço; os melhoramentos que consideram indispensáveis no serviço; as surpresas que considerou agradáveis e as surpresas desagradáveis que obteve durante a sua estadia no serviço. 
Para proceder à aplicação do formulário, foi feito o pedido de autorização prévia às 3 Instituições privadas de Imagiologia. Os pedidos para a realização do estudo foram aceites e autorizados. Os pacientes que fizeram parte do estudo foram elucidados acerca da fundamentação e dos objectivos do estudo, da confidencialidade e anonimato dos dados, fornecendo um consentimento autorizado sobre a sua participação.

Como a pesquisa combina métodos quantitativos e qualitativos, foram utilizadas frequências relativas, média e desvio padrão, Teste t, Análise da Variância a um factor (ANOVA I) e Análise de Conteúdo para as questões abertas, dividindo-se as respostas em várias categorias e subcategorias.

\section{Resultados}

Dos 213 inquiridos que compõem a amostra em estudo, 130 (61\%) pertencem ao sexo feminino e 83 (39\%) ao sexo masculino. Pela análise da tabela 1, verificamos que, e recorrendo ao $t$-test, existem diferenças significativas entre o sexo e as dimensões funcionais dos técnicos e médicos, com valores de $p$ $=0,000$ e $\mathrm{p}=0,004$, respectivamente, e entre o sexo e as amenidades, sendo o valor de $\mathrm{p}=\mathrm{o}, \mathrm{o} 37$, sendo que são os utentes do sexo feminino os que apresentam médias mais elevadas em todas as dimensões.

Tabela I - Médias do atendimento, dimensão funcional, dos técnicos e médicos, e amenidades segundo o sexo

\begin{tabular}{|c|c|c|c|c|c|c|}
\hline & Sexo & $\mathrm{N}$ & Média & $\mathrm{DP}$ & $t$ & $p$ \\
\hline \multirow[t]{2}{*}{ Atendimento } & Feminino & 130 & 23,7 & 6,9 & ।,836 & 0,068 \\
\hline & Masculino & 83 & 22,0 & 6,1 & & \\
\hline \multirow[t]{2}{*}{ D. Funcional Técnica } & Feminino & 130 & 22,4 & 10,8 & 5,345 & 0,000 \\
\hline & Masculino & 83 & 13,7 & 12,7 & & \\
\hline \multirow[t]{2}{*}{ D. Funcional Médica } & Feminino & 130 & 17,9 & 14,1 & 2,953 & 0,004 \\
\hline & Masculino & 83 & 12,1 & 13,5 & & \\
\hline \multirow[t]{2}{*}{ Amenidades } & Feminino & 130 & 21,0 & 5,3 & 2,096 & 0,037 \\
\hline & Masculino & 83 & 19,5 & 4,7 & & \\
\hline
\end{tabular}

Fonte: Resultados da pesquisa.

Relativamente à idade, constatamos que a média de idades dos 213 inquiridos é de 47 anos, sendo a idade mínima de 16 anos e a idade máxima de 85 anos. A maioria dos pacientes situa-se no grupo etário entre os 41 e os 50 anos, com uma percentagem de 33,8\%. Porém, não é de desprezar, a faixa etária dos 51 aos 60 anos, onde se situam $24,4 \%$ dos inquiridos. Quando analisamos as diferenças entre o grupo etário e o atendimento, dimensões funcionais e amenidades, verificámos que, e recorrendo ao teste Anova, apenas existem diferenças estatisticamente significativas, na dimensão amenidades ( $\mathrm{p}=\mathrm{o}, \mathrm{oo1}$ ). No entanto, é de assinalar que o grupo etário 81 é o que apresenta uma média mais elevada em relação às amenidades, sendo o grupo etário “31 - 40" o que apresenta maior média em relação ao atendimento e dimensão funcional dos técnicos.

Em relação à lista de espera para a realização dos exames, 59,6\% (127) dos inquiridos soube no próprio dia da marcação, o dia em que ia efectuar o exame. Mas no que se refere ao tempo que decorreu desde a marcação até ao dia do exame, 110 (51,6\%) inquiridos responderam que demorou "mais que uma semana", $53(24,9 \%)$ "uma semana", $37(17,4 \%)$ "mais que um dia" e apenas $13(6,1 \%)$ dos inquiridos dizem ter demorado "um dia". Quanto ao tempo que demorou a saber o dia do exame, 38 (17,8\%) dos inquiridos dizem ter demorado mais que um dia, $33(15,5 \%)$ uma 
semana e 15 (7\%) mais que uma semana. Existem diferenças estatisticamente significativas com o atendimento $(\mathrm{p}=\mathrm{o}, \mathrm{oo} 2)$, com a dimensão funcional dos médicos $(\mathrm{p}=\mathrm{o}, \mathrm{ooo})$ e com as amenidades $(\mathrm{p}=$ o,o3o). No entanto, referimos que o valor médio mais elevado no atendimento e amenidades enquadra-se nos inquiridos que referiram que esperaram só "um dia" pela marcação, mas nas dimensões funcionais, dos técnicos e dos médicos, os valores médios mais altos posicionam-se nos que referiram esperar "mais que um dia" e "mais que uma semana", respectivamente (cf tabela 2).

\section{Tabela 2 - Médias do atendimento, dimensão funcional dos técnicos e médicos e amenidades segundo o tempo}

de espera da marcação do exame

\begin{tabular}{|c|c|c|c|c|c|c|}
\hline & Tempo de espera para a marcação do exame & $\mathrm{N}$ & Média & $\mathrm{DP}$ & $\mathrm{F}$ & p \\
\hline \multirow[t]{4}{*}{ Atendimento } & Um dia & 13 & 29,2 & 7,9 & \multirow{4}{*}{5,314} & \multirow{4}{*}{0,002} \\
\hline & Mais que um dia & 37 & 24,1 & 6,7 & & \\
\hline & Uma semana & 53 & 23,1 & 6,5 & & \\
\hline & Mais que I semana & 110 & 21,9 & 6,2 & & \\
\hline Dimensão & Um dia & 13 & 18,5 & 14,3 & \multirow{4}{*}{1,035} & \multirow{4}{*}{0,378} \\
\hline \multirow[t]{3}{*}{ Funcional Técnica } & Mais que um dia & 37 & 22,2 & 12,1 & & \\
\hline & Uma semana & 53 & 18,7 & 12,4 & & \\
\hline & Mais que I semana & 110 & 18,2 & 12,1 & & \\
\hline Dimensão & Um dia & 13 & 13,8 & 15,9 & \multirow{4}{*}{10,244} & \multirow{4}{*}{0,000} \\
\hline \multirow[t]{3}{*}{ Funcional Médica } & Mais que um dia & 37 & 5,4 & 11,4 & & \\
\hline & Uma semana & 53 & 15,7 & 14,7 & & \\
\hline & Mais que I semana & 110 & 19,3 & 12,7 & & \\
\hline \multirow[t]{4}{*}{ Amenidades } & Um dia & 13 & 23,1 & 6,5 & \multirow{4}{*}{3,040} & \multirow{4}{*}{0,030} \\
\hline & Mais que um dia & 37 & 21,4 & 5,4 & & \\
\hline & Uma semana & 53 & 20,9 & 4,9 & & \\
\hline & Mais que I semana & 110 & 19,5 & 4,8 & & \\
\hline
\end{tabular}

Fonte: Resultados da pesquisa.

No que diz respeito às recomendações e sugestões dos pacientes acerca dos serviços privados de Imagiologia, há a saber que em relação ao facto de "recomendar o consultório a outra pessoa caso necessitasse de exames imagiológicos", as respostas foram claras: os inquiridos responderam "claro que sim" em 65,5\% e "provavelmente sim" em 34,3\%, sendo que apenas um (o, $5 \%$ ) dos inquiridos respondeu "provavelmente não". Relativamente ao facto de "voltar ao consultório caso necessitasse de exames imagiológicos", as respostas são de 6o,6\% para "claro que sim", 38,5\% para "provavelmente sim" e o,9\% para "provavelmente não". Em ambas as perguntas verifica-se que nenhum inquirido respondeu "claro que não".
Quanto às razões do regresso ou não ao consultório, as respostas foram maioritariamente positivas, destacando-se apenas 2 (o, $9 \%$ ) inquiridos que responderam que provavelmente não voltariam a frequentar o consultório. Para simplificar a avaliação dos resultados, dividimos a categoria principal (Razões porque voltaria ao consultório) em quatro subcategorias ou dimensões: relação e comunicação, organização dos serviços, informação e apoio, organização do espaço físico e continuidade e cooperação (cf tabela 3). A subcategoria "relação e comunicação" é a mais enumerada. No entanto, a unidade de registo "realização dos exames sempre no mesmo local", enquadrada na subcategoria "continuidade e cooperação", foi a que obteve mais unidades de enumeração (36). 
Tabela 3 - Distribuição das razões do cliente para voltar ao consultório

\begin{tabular}{|c|c|c|c|}
\hline Categoria & Subcategoria & Unidades de registo & Unidades de Enumeração (F) \\
\hline \multirow{13}{*}{$\begin{array}{l}\text { Razões porque } \\
\text { voltariam ao } \\
\text { consultório }\end{array}$} & \multirow[t]{3}{*}{ Relação e Comunicação } & Confiança nos profissionais de saúde & 23 \\
\hline & & Simpatia dos profissionais de saúde & 19 \\
\hline & & Competência dos profissionais de saúde & 28 \\
\hline & \multirow[t]{3}{*}{ Organização dos serviços } & Flexibilidade de horário & 23 \\
\hline & & Próximo do local de residência & 13 \\
\hline & & Bom atendimento & 21 \\
\hline & \multirow[t]{2}{*}{ Informação e apoio } & Fiabilidade dos resultados & 24 \\
\hline & & $\begin{array}{l}\text { Informação de outros profissionais de saúde } \\
\text { (médico assistente) }\end{array}$ & 15 \\
\hline & \multirow[t]{3}{*}{ Organização do espaço físico } & Ambiente agradável & 19 \\
\hline & & Bom aspecto & 18 \\
\hline & & Limpeza geral & 10 \\
\hline & \multirow[t]{2}{*}{ Continuidade e cooperação } & Acordo com todos os subsistemas & 10 \\
\hline & & Realização dos exames sempre no mesmo local & 36 \\
\hline
\end{tabular}

Fonte: Resultados da pesquisa.

Em relação aos aspectos que necessitam de ser melhorados no consultório, as respostas são muito variadas. Os resultados foram divididos em quatro subcategorias ou dimensões: relação e comunicação, organização dos serviços, informação e apoio e organização do espaço físico. Salientou-se em cada uma das subcategorias as unidades de registo mais enumeradas pelos inquiridos (cf tabela 4). A "diminuição da lista de espera”, "tempo de espera para a realização do exame" e "tamanho da sala de espera" foram as unidades de registo com mais unidades de enumeração, 44, 50 e 94, respectivamente.

\section{Tabela 4 - Distribuição da categoria "melhoramentos indispensáveis"}

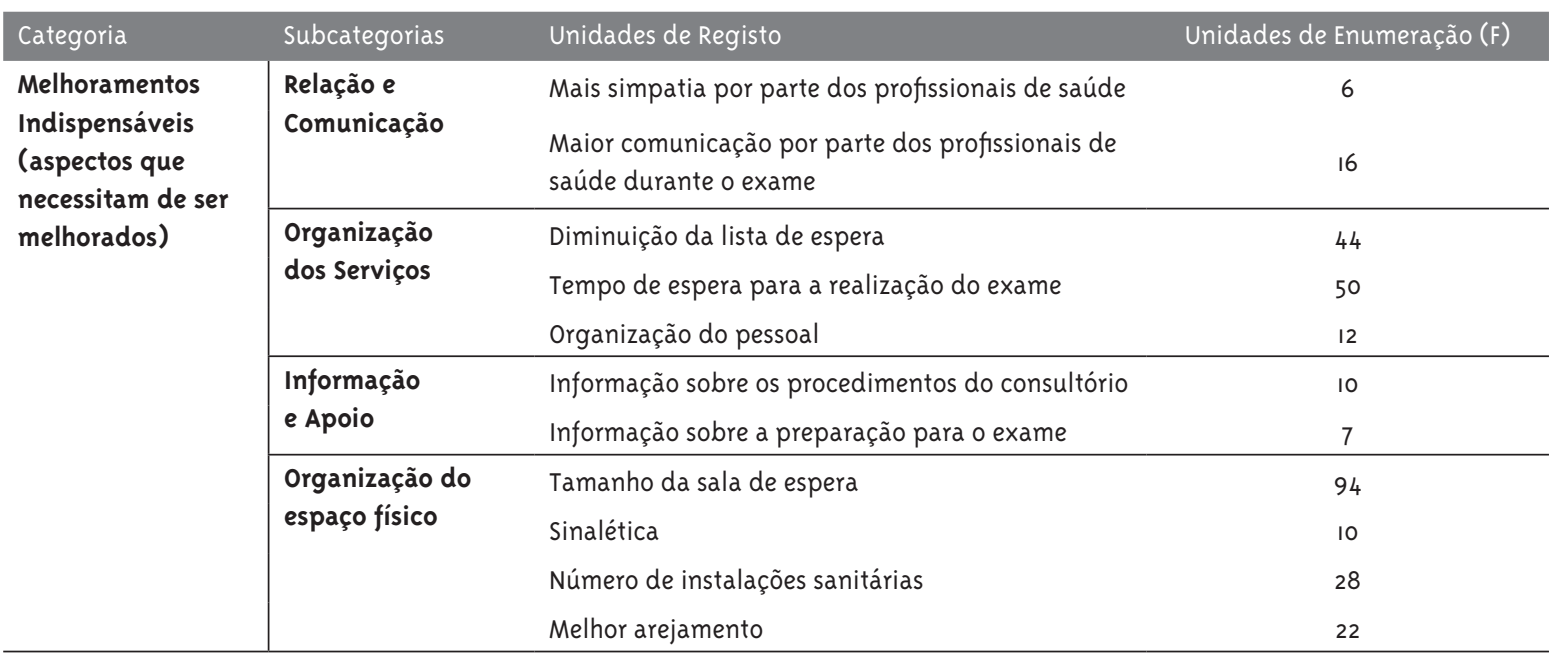


Quanto às surpresas agradáveis que os pacientes encontraram nos serviços de imagiologia, salientamos as seguintes: "privacidade" com $23 \%$, " simpatia e competência dos profissionais de saúde" com $21,1 \%$ e "resultado do exame" com $17,9 \%$ como as mais referidas. Destacamos ainda "limpeza geral" com 10,8\%, "rapidez no atendimento" com 13,6\% e 14,1\% referiram que "não se verificaram surpresas agradáveis”. No que diz respeito às surpresas desagradáveis, a maioria dos inquiridos assume o "tempo de espera para o exame" com 25,9\% e "falta de informação" com 16\%. Dentre os entrevistados, $16,5 \%$ responderam que "não houve surpresas desagradáveis".

\section{Discussão}

Os resultados do nosso estudo não se coadunam com os do estudo de Ferreira e Raposo (2006), ao afirmarem que as mulheres apresentam sempre uma satisfação inferior à dos homens. No entanto, Hall (1996), contraria esta linha de pensamento ao referir que os homens manifestam um nível de satisfação menor que os utentes do sexo feminino. Também Santos e Condeço (2002), vão ao encontro dos dados encontrados no nosso estudo na medida em que revelam existirem diferenças estatisticamente significativas em relação ao género no que respeita às instalações e higiene dos serviços.

No que se refere à idade, os resultados do nosso estudo estão em consonância com o estudo efectuado por Carvalho (2004), ao referir que os utentes mais idosos apresentam um maior nível de satisfação quando comparados com os utentes mais jovens, em relação às condições físicas de acolhimento. 0 estudo efectuado por Carvalho e Pacheco (2002), conclui que existe relação entre o aumento da idade e o grau de satisfação. Estes consideram que à medida que a idade aumenta, aumentam também os níveis de satisfação, uma vez que a população mais jovem mantém uma capacidade mais crítica e mais exigente com a vida, em geral, e com as instituições, em particular. Na mesma linha de pensamento, Ferreira e Raposo (2006) referem que os mais jovens estão sempre menos satisfeitos do que os mais idosos.

Relativamente aos tempos de espera, os nossos dados contrariam a conclusão de um estudo de
Franco e Florentim (2006), em que os utentes estão satisfeitos, em termos gerais, com os tempos de espera. Os resultados do nosso estudo confirmam o que refere Neto (2003), no seu estudo, que conclui que quanto menor o tempo de espera, maior a satisfação dos utentes. Estes dados replicam o observado no estudo de Ferreira (2001), em que os utentes se queixam do tempo de espera e sentem uma grande discrepância entre a altura da marcação e a efectivação da consulta.

Quanto ao recomendar e voltar ao consultório, os dados encontrados no nosso estudo, confirmam o que foi encontrado no estudo efectuado por Simões (2006), no qual os inquiridos manifestaram a sua plena satisfação, com uma percentagem de $100 \%$ nas perguntas de recomendação e intenção de voltar.

\section{Conclusões}

As organizações que prestam serviços de saúde têm como principal razão da sua existência as pessoas para as quais esses serviços são fornecidos. 0 facto de uma organização ter um utente como seu cliente exige que ela direccione as suas acções para responder ao seu anseio, de uma forma peculiar, pois o utente é um indivíduo física e/ou mentalmente comprometido no seu desenvolvimento pessoal ou nas suas relações com a sociedade.

$\mathrm{Na}$ busca por melhorias na gestão e pela vantagem competitiva, os gestores de organizações de saúde têm como ponto de partida questões sobre onde melhorar e que acções devem ser desencadeadas, ou seja, o que se deve fazer. Nesta busca pelas respostas, é importante que os clientes sejam a maior fonte de informações, pois são eles os utilizadores dos serviços e devem ser posicionados como o foco central no processo de decisão.

$\mathrm{O}$ que à primeira vista pode parecer muito complexo, não o é, se nos fixarmos no ponto fundamental que são os pacientes dos serviços de saúde como sendo clientes finais. Estes são o centro da definição e da prestação de serviços de saúde e dos cuidados sociais. São a razão de ser dos serviços e de toda a complexa rede interligada das unidades de saúde e de serviços sociais que foi construída para satisfazer as suas necessidades. Assim, o cliente mais importante dos serviços de saúde será sempre o 
paciente. Como tal, é necessário avaliar a sua satisfação ou insatisfação, em relação ao funcionamento organizacional, no sentido de melhorar os serviços prestados, porque a percepção dos pacientes é um precioso meio de gestão para a detecção de possíveis deficiências dos serviços prestados.

\section{Referências}

\section{ALMEIDA, H. A unidade funcional de saúde:} impacto nos barómetros do sistema de saúde português. Lisboa: Escola Nacional de Saúde Pública, 1999. XXVI Curso de Administração hospitalar 1996-1998.

BARROS, P. Economia da saúde: conceitos e comportamentos. Coimbra: Almedina, 2005.

CARVALHO, M. Satisfação dos utentes com os serviços de urgência. Porto: Serviço de Bioestatística e Informática Médica da Faculdade de Medicina do Porto, 2004.

CARVALHO, P.; PACHECO, S. Consulta de enfermagem de hipertensão: o grau de satisfação dos utentes. Nursing, Lisboa, v. 166, p. 21-26, 2002.

ESCOVAL, A. Sistemas de financiamento da saúde: análise e tendências. Lisboa: Associação Portuguesa de Economia da Saúde, 1999.

FERREIRA, G. Determinantes da satisfação dos utentes primários: o caso de Lisboa e Vale do Tejo. Revista de Saúde Pública, Lisboa, v. 2, p. 53-61, 2001.

FERREIRA, P.; RAPOSO, V. A governação em saúde e a utilização de indicadores de satisfação. Revista Portuguesa de Clínica Geral, Lisboa, v. 22, p. 285-296, 2006.

FRANCO, M.; FLORENTIM, R. A satisfação dos utentes em serviços de saúde: um estudo exploratório sobre o Departamento de Psiquiatria e Saúde Mental do Centro Hospitalar da Cova da Beira. Revista Portuguesa de Saúde Pública, Lisboa, v. 42, n. 1, p. 21-25, 2006.

GONÇALVES, M.; COSTA, J. O sistema de saúde português. In: NUNES, R. Política de saúde. Porto: Universidade Portucalense Infante D. Henrique: Artes Gráficas, 2003. p. 91-107.
HALL, M. Keys to patient satisfaction in the emergency department: results of a multiplefacility study. Hospital Health Service Administration, Chicago, v. 41, n. 4, p. 515-532, 1996.

HESPANHOL, A. A imagem dos serviços de saúde e dos médicos de família em Portugal. Revista Portuguesa Clínica Geral, Lisboa, v. 21, p. 185-191, 2004.

MEZOMO, J. Gestão da qualidade na saúde: princípios básicos. São Paulo: Manole, 2001.

NETO, A. Grau de satisfação do utente relativamente ao acolhimento proporcionado pelo enfermeiro no serviço de urgência. Servir, Lisboa, v. 51, n. 5, p. 214-227, 2003.

NUNES, R. Regulação da saúde. Porto: Vida Económica, 2005.

PINTO, S. Gestão dos serviços: a avaliação da qualidade. Lisboa: Verbo, 2003.

ROCHA, J. Gestão da qualidade: aplicação aos serviços públicos. Lisboa: Escolar, 2006.

ROSA, E. O agravamento do conflito público privado no Serviço Nacional de Saúde aumenta ineficiências e custos. Coimbra: Sindicato dos Médicos da zona Centro, 20o6. Disponível em: $<$ http//resistir.info/portugal/agravamento conflito_sns.html.>. Acesso em: 20 fev. 2009.

SAKELLARIDES, C. et al. O futuro do sistema de saúde português "Saúde 2015”. Lisboa: Escola Nacional de Saúde Pública, 2006.

SANTANA, P. Geografias da saúde do desenvolvimento: evolução e tendências em Portugal. Coimbra: Almedina, 2005.

SANTOS, E.; CONDEÇO, J. Avaliação do grau de satisfação dos dadores de sangue do Hospital Distrital de Chaves. ABO Revista de Medicina Transfusional, Lisboa, v. 11, p. 5-12, 2002.

SEPÚLVEDA, M. Satisfação dos utentes em relação à qualidade dos cuidados: consultas de ginecologia/obstetrícia e de cirurgia geral. Revista Portuguesa de Saúde Pública, Lisboa, v. 16 n. 4, p. 33-35, 1998. 
SIMÕES, C. O papel dos serviços de reabilitação

na satisfação dos seus utentes. Dissertação (Mestrado em Gestão)-Centro Regional das Beiras, Universidade Católica Portuguesa, Viseu, 2006.

VAVRA, T.; PRUDEN, D. Using aftermarketing to maintain a customer base. Discount

Merchandiser, Bristol, v. 35, n. 5, p. 86, 1995.

SIMOES, J. et al. Comissao para as sustentabilidade do financiamento do Servico Nacional de Saude: relatorio final. Lisboa:

Ministerio da Saude, 2007. Disponivel em: <http:// www.portaldasaude.pt/NR/rdonlyres/o5oCBoA27ACC-4975-A1E4-4312A1FBE12D/o/

RelatorioFinalComissaoSustentabilidade

FinanciamentoSNS.pdf>. Acesso em: 12 abr. 2009. 